\title{
Lateral and Vertical Heterostructures of Transition Metal Dichalcogenides
}

\author{
Mehmet Aras, ${ }^{\dagger}$ Çetin Kilıç, ${ }^{*}{ }^{\dagger}$ a and S. Ciraci*, ${ }^{*}$ \\ ${ }^{\dagger}$ Department of Physics, Gebze Technical University, 41400 Kocaeli, Turkey \\ ${ }^{\ddagger}$ Department of Physics, Bilkent University, 06800 Ankara, Turkey
}

Supporting Information

ABSTRACT: In this paper we investigate periodic lateral and vertical heterostructures of transition metal dichalcogenides (TMDs). Lateral heterostructures are constructed by the alternating metallic and semiconducting single-layer stripes of TMDs joined commensurately along their armchair edges and attain different states depending on the widths of constituents. While these heterostructures acquire a composite character with metallic state for narrow stripes, large stripes lead to the confinement of electronic states and function as metalsemiconductor junctions with a tunable Schottky barrier. The interface or boundary between constituent stripes has finite extension and allows charge transfer between them. On the other hand, the weak van der Waals interaction between layers sets the features of vertical heterostructures. Their interfaces are sharp: metal-semiconductor junction and Schottky barrier developed thereof can be induced even within a few layers. In the absence of dopants, we find minute charge transfer across the interface with negligible band bending in vertical heterostructures. The $\delta$-doping of the semiconducting constituent by the metallic one forms strictly two-dimensional metallic electrons in a three-dimensional layered semiconductor and leads to crucial directionality effects and quantization of conductance. Our work unveils significant differences between lateral and vertical heterostructures.

\section{INTRODUCTION}

In recent years, a variety of heterostructures have been fabricated from the vertical or lateral combination of singlelayer (SL) or multilayer (ML) transition metal dichalcogenides (TMDs) with other TMDs or with other two-dimensional (2D) materials such as graphene. Prototype devices based on these TMD-based heterostructures have been assembled for various electronic, optoelectronic, and photovoltaic applications; see, e.g., refs 1 and 2 for recent reviews. For example, both vertical and lateral heterostructures comprising semiconductor/semiconductor junctions have been used as active components in $\mathrm{p}-\mathrm{n}$ diodes, ${ }^{3-16}$ photodiodes, ${ }^{11,12}$ photodetectors, ${ }^{6,9,17}$ and field effect transistors (FETs), ${ }^{6,7,18}$ which were also the main subject of numerous theoretical studies. ${ }^{19-26}$ In contrast, TMD-based heterostructures comprising metal/semiconductor junctions have been studied to a much lesser extent, albeit their unusual electronic properties and important device capacities thereof have been heralded by early experimental $^{27,28}$ and theoretical ${ }^{29-31}$ studies. It should also be pointed out that using metallic SL TMDs stacked with semiconducting SL TMDs (as in vertical heterostructures) has been explored ${ }^{32,33}$ as a viable strategy for achieving an efficient and stable electrical contact between 2D semiconductors and metal electrodes.
This paper presents a theoretical study of lateral (in-plane) and vertical (van der Waals) heterostructures made of nearly lattice matched metallic (SL $\mathrm{NiTe}_{2}$ ) and semiconducting (SL $\left.\mathrm{MoTe}_{2}\right)$ TMDs. Our objectives are to reveal the effects of size and dimensionality of constituents on the electronic structure of these heterostructures and composite materials. The periodically repeating, lateral, and commensurate junctions of semiconducting $\mathrm{MoTe}_{2}$ and metallic $\mathrm{NiTe}_{2}$ stripes along their armchair edge make a class of new materials. These armchair edged lateral heterostructures we treat in this paper are specified as A: $\left(\mathrm{MoTe}_{2}\right)_{p} /\left(\mathrm{NiTe}_{2}\right)_{q}$ with $1 \leq p$ and $q \leq 10$, where $p$ and $q$ are the numbers of formula units in the lateral primitive unit cell. Depending on the values of $p$ and $q$, these lateral structures display a number of properties ranging from composite metals to metal-semiconductor junction having a Schottky barrier, which can be tuned by varying $p$.

The situation in vertical heterostructures, which are constructed from the stacking of SL $\mathrm{MoTe}_{2}$ and $\mathrm{NiTe}_{2}$ layers and are specified as $\mathrm{V}:\left(\mathrm{MoTe}_{2}\right)_{p} /\left(\mathrm{NiTe}_{2}\right)_{q}$ with $1 \leq p$ and $q \leq$ 5 , is, however, different. Because of weak van der Waals (vdW) interlayer interaction, the coupling between adjacent TMDs

Received: August 18, 2017

Revised: December 26, 2017

Published: January 5, 2018 
stacked vertically is rather weak, and it has small but crucial effects on the electronic structure as will be discussed in the forthcoming sections. This enhances confinements of electrons in different constituents. Despite the weak interlayer interaction, the resulting electronic structures in composite vertical structures with very thin as well as wide alternation of constituents are of current interest.

SL TMD constituents used in this study have been synthesized long ago. ${ }^{34}$ Dynamical and thermal stability analysis has demonstrated that many freestanding SL TMDs (including $\mathrm{MoTe}_{2}$ and $\mathrm{NiTe}_{2}$ ) with hexagonal and trigonal structures are stable. ${ }^{35}$ Vertical stacks of TMDs and their heterostructures were also found to be stable. ${ }^{7}$ The stability of freestanding layers is clearly enhanced if they were grown on specific substrates. In this respect, the stability of bilayers and multilayer stacks is also enhanced. Recent theoretical stability analysis ${ }^{29,35}$ and extensive structure optimization calculations carried out here as well as recent experimental studies ${ }^{7,27,28,33,36-38}$ indicate that the vertical and lateral heterostructures treated in the present study are stable and can be fabricated. It should also be pointed out that lateral homojunctions combining hexagonal (semiconducting) and monoclinic (metallic) phases of $\mathrm{MoTe}_{2}$ have already been fabricated via laser-induced phase patterning. ${ }^{27}$ Furthermore, bilayer (vertical) heterostructures composed of $\mathrm{MoS}_{2}$ and $\mathrm{MoTe}_{2}$ monolayers were successfully prepared by a (mechanical) dry transfer process. ${ }^{33}$

The important findings of the present study are summarized as follows: (i) The lateral heterostructure is a metal for narrow constituent stripes, but the separation of semiconducting and metallic regions and the junction formation occur only after a threshold widths of constituents. The Fermi level is pinned and the Schottky barrier is formed by metallic states of the $\mathrm{NiTe}_{2}$ side, which decay into the semiconducting $\mathrm{MoTe}_{2}$ side. The charge transferred this way results in a linear bending of average potential at the boundary region. (ii) The energies of band edges in the semiconducting stripe can be modified by confinement effects. (iii) In vertical heterostructures, the separation of metal-semiconductor zones in direct space is complete even for two layers of constituents. Because of minute charge transfer at the undoped junction, the band bending at the interface is negligible. The fundamental band gap of the semiconducting side is indirect and is also smaller than that of SL $\mathrm{MoTe}_{2}$ constituent. (iv) A strictly 2D metallic system confined to a single layer can be achieved when an $\mathrm{SL} \mathrm{NiTe}$ is inserted into the $\mathrm{MoTe}_{2}$ vertical stack, which can exhibit exceptional behaviors.

\section{MODELS AND COMPUTATIONAL DETAILS}

Our atomistic models of the lateral and vertical heterostructures are described in Figure 1 . Along the $x$-direction of the lateral ones in Figure 1a,b the constituent stripes, SL $\mathrm{MoTe}_{2}$ and $\mathrm{NiTe}_{2}$, extend to infinity (without any edge); the $y$-direction is perpendicular to the armchair edges, along which two different stripes repeat alternatingly and periodically. In the $x y$-plane, the heterostructure has a $2 \mathrm{D}$ rectangular lattice and each constituent consists of three parallel atomic planes, where the plane of metal atoms ( $\mathrm{Mo}$ or $\mathrm{Ni}$ ) is capped by two Te atomic planes. The interfaces (or boundaries) between two commensurately joined constituent stripes, $\mathrm{I}_{1}$ and $\mathrm{I}_{2}$, are onedimensional. The lateral heterostructures are repeated periodically along the $z$-direction with a vacuum spacing of $20 \AA$ in a three-dimensional (3D) orthorhombic lattice. Small lattice mismatches between constituents induce inhomogeneous small

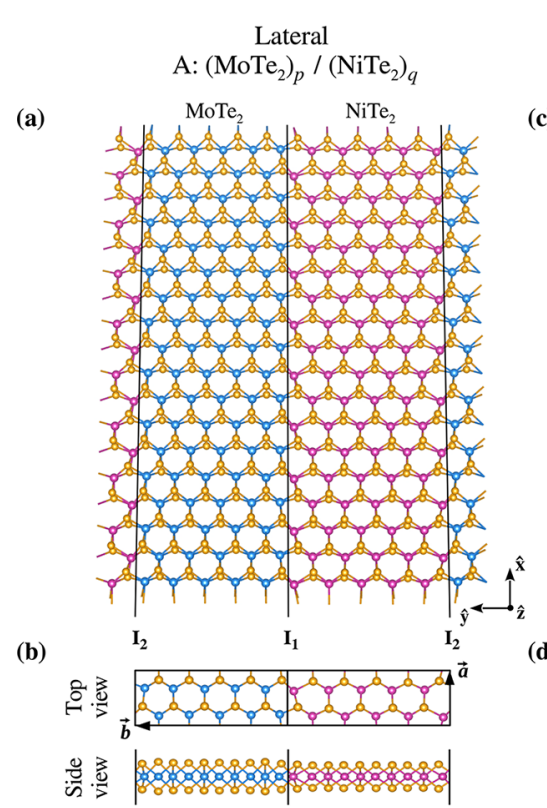

Vertical

$\mathrm{V}:\left(\mathrm{MoTe}_{2}\right)_{p} /\left(\mathrm{NiTe}_{2}\right)_{q}$

(c)

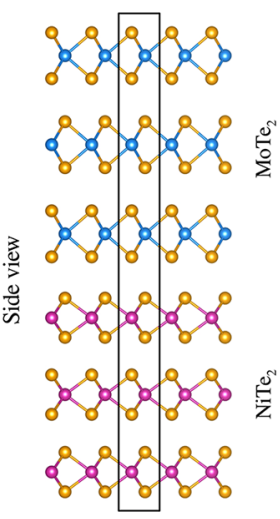

(d)

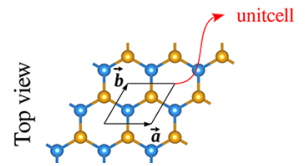

Figure 1. (a) Top and (b) side views of atomistic model of SL, lateral heterostructure A: $\left(\mathrm{MoTe}_{2}\right)_{p} /\left(\mathrm{NiTe}_{2}\right)_{q}$ with $p=10$ and $q=10$. Blue, magenta, and gold balls indicate $\mathrm{Mo}, \mathrm{Ni}$, and $\mathrm{Te}$ atoms, respectively. Two-dimensional rectangular unit cell with Bravais lattice vectors a and $\mathbf{b}$ is also shown. $\mathrm{I}_{1}$ and $\mathrm{I}_{2}$ are interfaces (or boundaries) between different constituent TMD stripes along the armchair edges. (c) Side view of the vertical heterostructure V: $\left(\mathrm{MoTe}_{2}\right)_{p} /\left(\mathrm{NiTe}_{2}\right)_{q}$ with $p=3$ and $q=3$. (d) Top view. Three-dimensional hexagonal unit cell is delineated in side and top views.

strains in the heterostructures, which can easily be compensated.

Vertical heterostructures are produced by layer-by-layer stacking of 2D SL constituents, which interact weakly with adjacent layers. A vertical stack of $p$ layers of one constituent is followed by a stack of $q$ layers of the other constituent as shown in Figure 1c. This stacking sequence repeats periodically and continuously with a 3D hexagonal lattice.

We carried out spin-polarized density-functional calculations within either the local density approximation (LDA) using the functional of Ceperley and Alder ${ }^{39}$ or the generalized gradient approximation (GGA) using the functional of Perdew, Burke, and Ernzerhof ${ }^{40}$ (PBE) combined with the semiempirical dispersion (D3) correction. ${ }^{41}$ We used the VASP code, ${ }^{42}$ and employed the projector augmented wave (PAW) method. ${ }^{43,44}$ The $3 \mathrm{~d}$ and $4 \mathrm{~s}, 4 \mathrm{~d}$ and $5 \mathrm{~s}$, and $5 \mathrm{~s}$ and $5 \mathrm{p}$ states were treated as valence states for $\mathrm{Ni}, \mathrm{Mo}$, and $\mathrm{Te}$ atoms, respectively. Bloch states of electrons are expressed by plane wave basis sets with a kinetic energy cutoff of $400 \mathrm{eV}$. Lateral heterostructures are treated using the supercell geometry with a sufficient vacuum spacing between layers to prevent coupling between them. The vacuum spacing of $20 \AA$ is determined by convergence tests. For example, the energy difference between 25 and $20 \AA$ vacuum spacings is found to be only $0.14 \mathrm{meV} /$ atom. Brillouin zones were sampled using the Monkhorst-Pack scheme ${ }^{45}$ where the $k$-point mesh is adjusted according to the size of cells in the direct space; see Tables S1-S3 in Supporting Information. All structures treated in this study are fully optimized; we performed concurrent relaxations of the lattice parameters and ionic positions until the maximum values of residual forces on atoms were reduced to be smaller than 0.01 $\mathrm{eV} / \AA$. The convergence criterion for the electronic self- 
consistency was set up to $10^{-6} \mathrm{eV}$ (in structure optimizations) and $10^{-8} \mathrm{eV}$ (in electronic structure calculations). The definition of the cohesive energy $E_{\mathrm{c}}$ and formation energy $E_{\mathrm{f}}$, which are used to specify the energetics, are presented in the Supporting Information. We note that cohesive energy is given relative to constituent atoms, and $E_{\mathrm{c}}$ indicates cohesion. The formation energy is given relative to $3 \mathrm{D}$ layered bulk parent crystals, which constitute global minima. Accordingly, heterostructures with slightly negative formation energies are stable in local minima on the Born-Oppenheimer surface.

Before we start to study heterostructures, we note that whereas $2 \mathrm{D} \mathrm{SL} \mathrm{NiTe}$ is a nonmagnetic metal, 2D SL MoTe $\mathrm{Me}_{2}$ is a semiconductor with a direct band gap. Earlier it was shown that LDA predicts the fundamental band gaps of SL TMDs close to the measured values. ${ }^{46}$ Also, earlier experimental and LDA band gaps of TMDs such as $\mathrm{MoS}_{2}, \mathrm{MoSe}_{2}, \mathrm{WS}_{2}$, and $\mathrm{WSe}_{2}$ were shown to be in agreement. ${ }^{47}$ The band gap of SL $\mathrm{MoTe}_{2}$ is calculated using LDA to be direct, and $E_{\mathrm{g}}=1.25 \mathrm{eV}$. This value is in agreement with the experimental value of 1.10 $\mathrm{eV}^{48}$ This band gap increased to only $1.34 \mathrm{eV}$ by applying the correction using hybrid functionals. ${ }^{49,50}$ On the other hand, the band gap decreased slightly to $1.15 \mathrm{eV}$ in calculations using PBE-D3. Given this relatively small variation in the band gap, the lateral heterostructures consisting of a single layer are treated within the LDA. However, the van der Waals (vdW) interaction is taken into account for the vertical heterostructures through the PBE-D3 approach. ${ }^{41}$ We did not apply the $G W$ correction, since it is not feasible computationally for the large systems we are treating and also is known to overestimate the band gaps of SL TMDs. ${ }^{46}$

In Tables 1 and 2 we summarize optimized values of cohesive energy, formation energy, optimized lattice constants, and

Table 1. Optimized Values of Lateral Heterostructure, A: $\left(\mathrm{MoTe}_{2}\right)_{p} /\left(\mathrm{NiTe}_{2}\right)_{q}$ for $1 \leq p=q \leq 10$, and Values of Average Cohesive Energy, $\overline{\boldsymbol{E}}_{\mathfrak{c}}$, Average Formation Energy $\overline{\boldsymbol{E}}_{\mathrm{f}}$, Two-Dimensional Rectangular Lattice Constants $a$ and $b$, and Fundamental Band Gap, $E_{\mathrm{g}}$

$\begin{array}{crrrrr}\text { composite }(p / q) & \bar{E}_{\mathrm{c}}(\mathrm{eV}) & \bar{E}_{\mathrm{f}}(\mathrm{eV}) & a(\AA) & b(\AA) & E_{\mathrm{g}}(\mathrm{eV}) \\ \mathrm{A}:(1 / 1) & 13.780 & -0.707 & 5.966 & 3.444 & \\ \mathrm{~A}:(2 / 2) & 13.893 & -0.594 & 6.083 & 7.025 & \\ \mathrm{~A}:(3 / 3) & 14.049 & -0.438 & 6.054 & 10.486 & \\ \mathrm{~A}:(4 / 4) & 14.119 & -0.369 & 6.090 & 14.065 & \\ \mathrm{~A}:(5 / 5) & 14.128 & -0.360 & 6.067 & 17.515 & 0.426 \\ \mathrm{~A}:(6 / 6) & 14.143 & -0.344 & 6.098 & 21.123 & 0.778 \\ \mathrm{~A}:(7 / 7) & 14.151 & -0.336 & 6.089 & 24.608 & 0.962 \\ \mathrm{~A}:(8 / 8) & 14.158 & -0.329 & 6.073 & 28.050 & 1.053 \\ \mathrm{~A}:(9 / 9) & 14.164 & -0.323 & 6.076 & 31.572 & 1.124 \\ \mathrm{~A}:(10 / 10) & 14.172 & -0.315 & 6.074 & 35.065 & 1.173\end{array}$

fundamental band gaps of lateral and vertical heterostructures A: $\left(\mathrm{MoTe}_{2}\right)_{p} /\left(\mathrm{NiTe}_{2}\right)_{q}$ and $\mathrm{V}:\left(\mathrm{MoTe}_{2}\right)_{p} /\left(\mathrm{NiTe}_{2}\right)_{q}$, respectively. Values of $3 \mathrm{D}$ crystals, bilayers, trilayers, and monolayers are presented as Supporting Information.

\section{RESULTS}

Lateral Heterostructures. The electronic structure of 2D $\mathrm{SL} \mathrm{MoTe}_{2}$ and $\mathrm{NiTe}_{2}$ as well as the energetics and thermal stability of zigzag or armchair edged lateral heterostructures were discussed in our previous study. ${ }^{29}$ In the present study we consider features which were not treated earlier. Moreover, we
Table 2. Optimized Values of Vertical Heterostructure, V: $\left(\mathrm{MoTe}_{2}\right)_{p} /\left(\mathrm{NiTe}_{2}\right)_{q}$, and Values of Average Cohesive Energy, $\bar{E}_{\mathrm{c}}$, Average Formation Energy $\overline{\boldsymbol{E}}_{\mathrm{f}}$, Superlattice Constants $a=b, c$, Interface Spacing $d_{\text {int }}$ and Fundamental Indirect Band Gap, $E_{\mathrm{g}}$

\begin{tabular}{ccccccc}
$\begin{array}{c}\text { composite } \\
(p / q)\end{array}$ & $\bar{E}_{\mathrm{c}}(\mathrm{eV})$ & $\bar{E}_{\mathrm{f}}(\mathrm{eV})$ & $\begin{array}{c}a=b \\
(\AA)\end{array}$ & $c(\AA)$ & $d_{\text {int }}(\AA)$ & $\begin{array}{c}E_{\mathrm{g}} \\
(\mathrm{eV})\end{array}$ \\
$\mathrm{V}:(1 / 1)$ & 11.988 & -0.075 & 3.589 & 12.797 & 3.108 & \\
$\mathrm{~V}:(2 / 2)$ & 12.012 & -0.051 & 3.581 & 25.910 & 3.227 & \\
$\mathrm{~V}:(3 / 3)$ & 12.019 & -0.044 & 3.579 & 38.887 & 3.271 & 0.641 \\
$\mathrm{~V}:(4 / 4)$ & 12.023 & -0.040 & 3.583 & 51.667 & 3.203 & 0.657 \\
$\mathrm{~V}:(5 / 1)$ & 12.734 & -0.032 & 3.528 & 41.192 & 3.306 & 0.722 \\
$\mathrm{~V}:(1 / 5)$ & 11.328 & -0.033 & 3.651 & 36.239 & 3.105 & \\
\hline
\end{tabular}

contrast these features with those of the vertical heterostructures.

The armchair edged lateral heterostructures A: $\left(\mathrm{MoTe}_{2}\right)_{p} /$ $\left(\mathrm{NiTe}_{2}\right)_{q}$ have large cohesive energies in the range of $E_{\mathrm{c}}=14$ $\mathrm{eV}$ per formula unit, but negative formation energies in the range of $E_{\mathrm{f}}=-0.4 \mathrm{eV}$. However, the growth of a heterostructure from two freestanding constituent stripes is favored, since the formation of junction is exothermic due to saturation of dangling bonds at the boundaries. The electronic structures of these heterostructures made of the periodic junctions of semiconducting and metallic stripes of $\mathrm{MoTe}_{2}$ and $\mathrm{NiTe}_{2}$ of different widths exhibit diverse features. The most interesting feature is that they are metallic in both momentum and direct space for small $p$ and $q$; these heterostructures can be considered as a composite structure or a special alloy. However, electronic states are confined in different constituent stripes as $p$ and $q$ increase and constituent stripes start to display the electronic properties of their parent 2D TMDs. Namely, the heterostructure changes from metal to metal-semiconductor junction, as indicated in Figure 2. For $p=q \geq 5$, a band gap

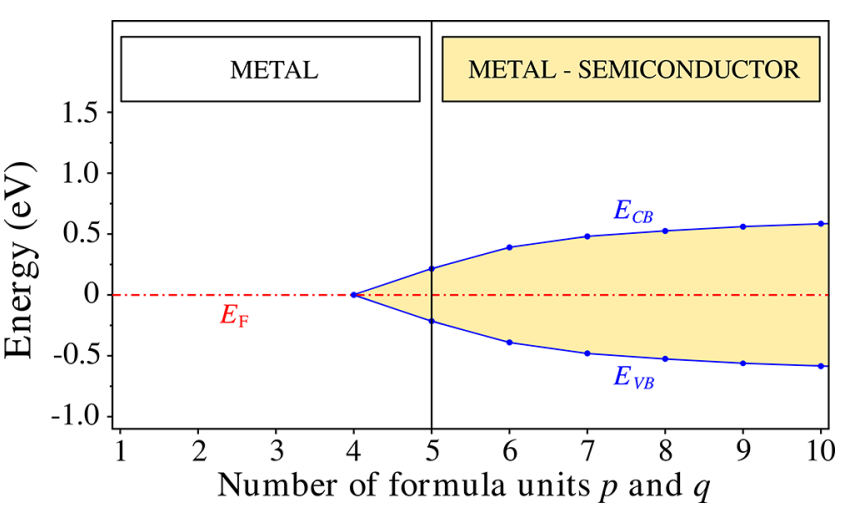

Figure 2. Transition from metal to metal-semiconductor junction with increasing $p=q$ in A: $\left(\mathrm{MoTe}_{2}\right)_{p} /\left(\mathrm{NiTe}_{2}\right)_{q}$ heterostructure. The band gap between the conduction $E_{\mathrm{CB}}$ and valence band $E_{\mathrm{VB}}$ edges at the center of $\mathrm{MoTe}_{2}$ is shaded. The zero of energy is set at the Fermi level $E_{\mathrm{F}}$.

starts to open in the $\mathrm{MoTe}_{2}$ stripe, which is enlarged as $p$ increases, while $\mathrm{NiTe}_{2}$ remains a metal. This band gap in the $\mathrm{MoTe}_{2}$ stripe constitutes a potential barrier for the metallic states of $\mathrm{NiTe}_{2}$, and causes them to be confined between two $\mathrm{MoTe}_{2}$ stripes. Hence, the heterostructure starts to function as a metal-semiconductor junction for $p=q \geq 5$, but the semiconducting stripe assumes the electronic properties of $2 \mathrm{D}$ $\mathrm{MoTe}_{2}$ for $p \sim 10$. The charge can be transferred across the 
A: $\left(\mathrm{MoTe}_{2}\right)_{1} /\left(\mathrm{NiTe}_{2}\right)_{1}$
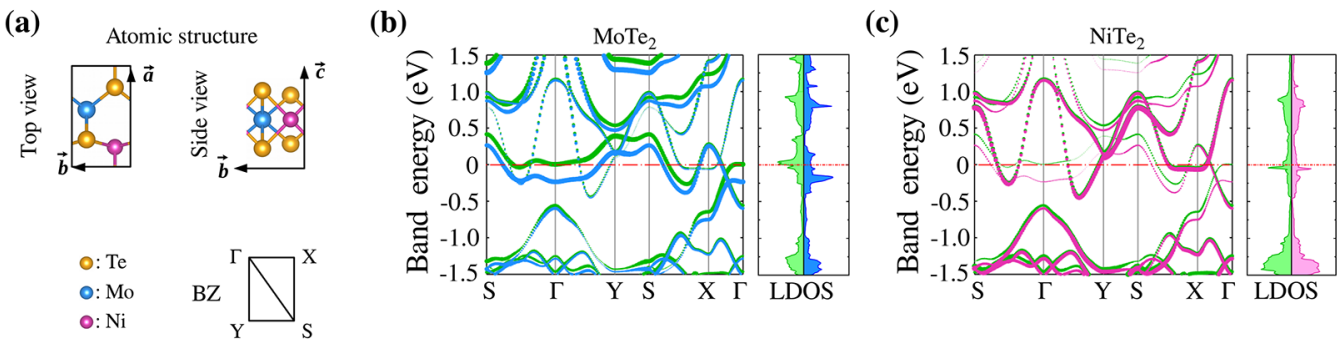

\section{A: $\left(\mathrm{MoTe}_{2}\right)_{5} /\left(\mathrm{NiTe}_{2}\right)_{5}$}
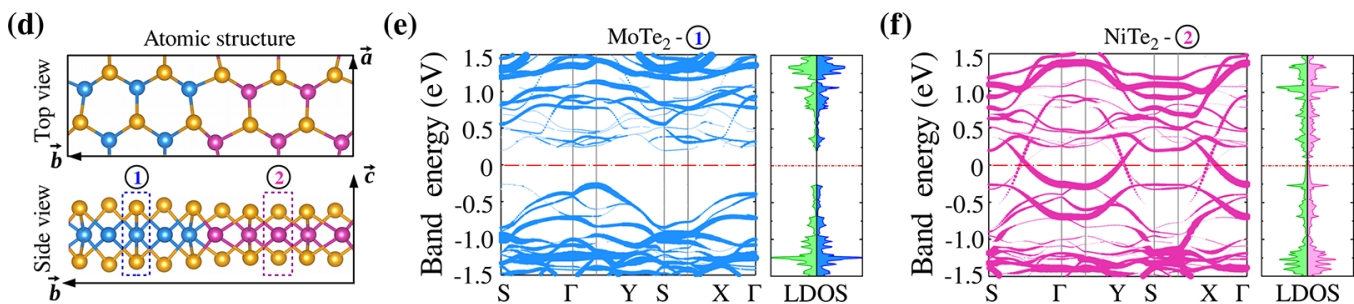

Figure 3. A: $\left(\mathrm{MoTe}_{2}\right)_{1} /\left(\mathrm{NiTe}_{2}\right)_{1}$ lateral heterostructure. (a) Unit cell with $2 \mathrm{D}$ rectangular lattice and corresponding Brillouin zone, BZ. (b) Bands projected to the $\mathrm{Te}-\mathrm{Mo}-\mathrm{Te}$ unit in the unit cell and corresponding asymmetric spin-polarized projected densities of states. (c) Bands projected to the $\mathrm{Te}-\mathrm{Ni}-\mathrm{Te}$ unit in the unit cell with spin-polarized projected densities of states. (d-f) Same for A: $\left(\mathrm{MoTe}_{2}\right)_{5} /\left(\mathrm{NiTe}_{2}\right)_{5}$. Bands are projected to the $\mathrm{Te}-\mathrm{Mo}-\mathrm{Te}$ and $\mathrm{Te}-\mathrm{Ni}-\mathrm{Te}$ units in the labeled unit cells. Zero of energy is set to the common Fermi level.

boundaries $I_{1}$ and $I_{2}$, whereby the edges of the conduction and valence bands in direct space are bent.

The confinement of electrons in adjacent stripes leading to a metal-semiconductor transition through phase separation in direct space and eventually the formation of Schottky barrier will now be discussed in detail. In Figure 3, the unit cell, Brillouin zone (BZ), energy bands projected to $\mathrm{Te}-\mathrm{Mo}-\mathrm{Te}$ and $\mathrm{Te}-\mathrm{Ni}-\mathrm{Te}$ units in the heterostructures, and corresponding projected densities of states of $\mathrm{A}:\left(\mathrm{MoTe}_{2}\right)_{p} /\left(\mathrm{NiTe}_{2}\right)_{q}$ for $p$ $=q=1$ and $p=q=5$ are shown. The energy bands projected to $\mathrm{Te}-\mathrm{Mo}-\mathrm{Te}$ and $\mathrm{Te}-\mathrm{Ni}-\mathrm{Te}$ units are of particular importance, since they show the contribution of these units to the states of any band.

For $p=q=1$, both $\mathrm{Te}-\mathrm{Mo}-\mathrm{Te}$ and $\mathrm{Te}-\mathrm{Ni}-\mathrm{Te}$ units contribute to the density of states (DOS) at the Fermi level and attribute a metallic character to the heterostructure. Additionally, the spin-polarized DOS is asymmetric at the $\mathrm{MoTe}_{2}$ side, but the resulting net magnetic moment is negligible. The situation for $\mathrm{A}:\left(\mathrm{MoTe}_{2}\right)_{5} /\left(\mathrm{NiTe}_{2}\right)_{5}$, however, is rather different. The contribution of a $\mathrm{Te}-\mathrm{Mo}-\mathrm{Te}$ unit at the center of the $\mathrm{MoTe}_{2}$ stripe to the bands at the Fermi level ceases, while the contribution of a $\mathrm{Te}-\mathrm{Ni}-\mathrm{Te}$ unit at the Fermi level continues. This is the initial stage for the onset of the metalsemiconductor transition.

The electronic phase separation and band lineup leading to Schottky barrier formation is completed in A: $\left(\mathrm{MoTe}_{2}\right)_{10} /$ $\left(\mathrm{NiTe}_{2}\right)_{10}$, where the band gap at the $\mathrm{MoTe}_{2}$ stripe approaches the value of the fundamental band gap of $2 \mathrm{D} \mathrm{SL} \mathrm{MoTe}_{2}$ as shown in Figure 4. The plane averaged electronic potential energy and its average value $\bar{V}(y)$ shown in Figure $4 \mathrm{~b}$ are different in different stripes. $\bar{V}(y)$ is nearly flat inside each stripe, but its value changes smoothly and linearly across the boundary. This is in compliance with the Schottky-Mott theory. $^{51,52}$

In Figure 4c, DOSs projected to different $\mathrm{Te}$-cation-Te units indicated by the numerals are shown. The band gap is opened inside the central $\mathrm{MoTe}_{2}$ stripe, but it is closed by approaching the boundaries. However, the $\mathrm{NiTe}_{2}$ stripe is metallic with finite DOS at the common Fermi level. This is the demonstration of a metal-semiconductor transition, whereby a totally metallic heterostructure $\mathrm{A}:\left(\mathrm{MoTe}_{2}\right)_{p} /\left(\mathrm{NiTe}_{2}\right)_{q}$ with $p=$ $q \leq 4$ changes to a semiconductor in the $\mathrm{MoTe}_{2}$ side and forms a Schottky barrier for $p=q=10$. This transition occurs with the confinement of electronic states to one of the constituents. At the end each stripe transforms gradually to its parent 2D SL structure with increasing $p$ and $q$. Owing to the symmetry of the boundaries, $\bar{V}(y)$ is not tilted inside the semiconducting side of the heterostructure.

To determine the Schottky barrier, we first obtain the energy band diagram in direct space. To this end, the energies of the valence band edge, $E_{\mathrm{VB}}$, and the conduction band edge, $E_{\mathrm{CB}}$, relative to $\bar{V}(y)$ are retrieved from SL 2D $\mathrm{MoTe}_{2}$. In spite of the fact that this is a rather rigorous method for very wide $\mathrm{MoTe}_{2}$ stripes, $E_{\mathrm{VB}}$ and $E_{\mathrm{CB}}$ can conveniently be determined directly from the local density of state (LDOS) by projecting the DOS to the $\mathrm{Te}-\mathrm{Mo}-\mathrm{Te}$ unit at the center of the stripe as illustrated in Figure 4c. This way effects due to confinement of carriers in the stripe are taken into account. Then, following the methods developed earlier for 3D junctions, ${ }^{53,54}$ and by marking these band edges relative to $\bar{V}(y)$, one can draw the band diagram in direct space. Here the premise is that the energies of $E_{\mathrm{VB}}$ and $E_{\mathrm{CB}}$ will remain invariant across the $\mathrm{MoTe}_{2}$ stripe. Accordingly, owing to the linear variation of $\bar{V}(y)$ near boundaries, the band edges are bent at the interface. By definition, the Schottky barrier energy $E_{\mathrm{S}}$ is the energy from the common Fermi level to $E_{\mathrm{CB}}$ at the interface, which is taken to be sharp. Based on this construction, the Schottky barrier is calculated to be $E_{\mathrm{S}}=1.03$ $\mathrm{eV}$. We note that the value of $E_{\mathrm{S}}$ depends on the widths of stripes, or $p$ and $q$, but it converges to a value for a very wide stripe.

The physical explanation of pinning of the Fermi level and bending of bands is as follows: Upon the opening of a band gap inside the $\mathrm{MoTe}_{2}$ stripe, metallic $\mathrm{NiTe}_{2}$ states have to decay inside $\mathrm{MoTe}_{2}$ when their energies coincide with the gap. This way, excess electronic charge is accumulated at the $\mathrm{MoTe}_{2}$ side close to the boundary, which in turn, bends $\bar{V}(y)$ near the 
(a)

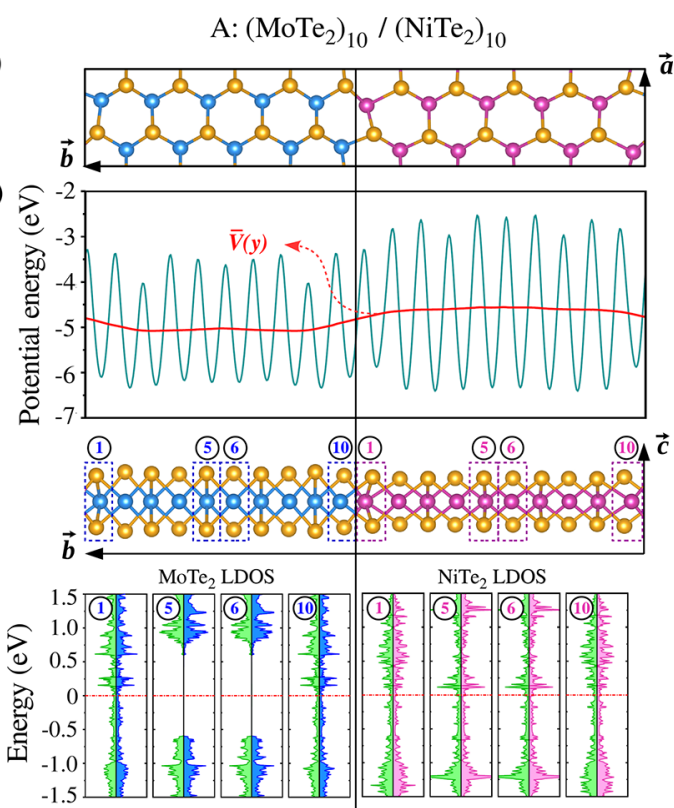

(d)

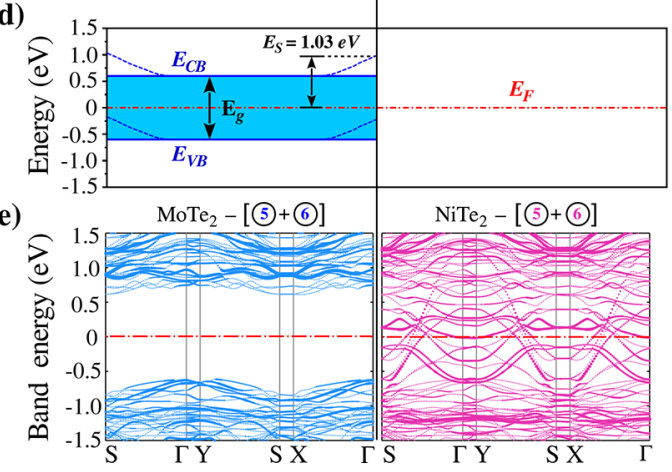

Figure 4. Heterostructure A: $\left(\mathrm{MoTe}_{2}\right)_{10} /\left(\mathrm{NiTe}_{2}\right)_{10}$. (a) Unit cell with lattice vectors. (b) Plane averaged electronic potential energy and its average along $y$-direction, $\bar{V}(y)$. (c) Spin-polarized densities of states projected to $\mathrm{Te}-\mathrm{Mo}-\mathrm{Te}$ and $\mathrm{Te}-\mathrm{Ni}-\mathrm{Te}$ units in the labeled unit cells. (d) Formation of metal (in $\mathrm{NiTe}_{2}$ stripe)-semiconductor (in $\mathrm{MoTe}_{2}$ stripe) junction with band lineup and Schottky barrier. (e) Bands projected to the $\mathrm{Te}-\mathrm{Mo}-\mathrm{Te}$ and $\mathrm{Te}-\mathrm{Ni}-\mathrm{Te}$ units in the unit cells specified in (c).

boundaries. Metallic states of $\mathrm{NiTe}_{2}$ decaying toward $\mathrm{MoTe}_{2}$ and pinning the Fermi level is reminiscent of the metal induced states (MIGS) proposed for 3D bulk metal-semiconductor junctions. ${ }^{55,56}$

At this point another point worth emphasizing concerns the width of the interface: Our results show that the boundary or interface between metal and insulator is not sharp; a distance corresponding to a few $\mathrm{MoTe}_{2}$ units is needed at each boundary to recover the semiconducting side of the heterostructure. This situation marks the dramatic difference between present 2D lateral and 3D bulk metal-semiconductor junctions. In this respect, crucial aspects one should take into account are the dimensionality, thickness, and width of the inplane lateral structure. Our calculations indicate the following crucial features: (i) The boundary region between metallic and semiconducting stripes cannot be sharp, but has a finite extension. (ii) The energy band diagram in the direct space cannot be uniform across the semiconducting stripe; the band gap can be closed in the boundary region because of metallic states penetrating the semiconducting side. Under these circumstances, the Schottky barrier of in-plane, lateral heterostructures can be determined directly from LDOS calculated at the atoms across the semiconducting stripe. One should keep in mind that an uncertainty is involved in determining band edges from LDOS in this direct method. In Figure $4 \mathrm{e}$ the Schottky barrier determined from LDOS appears to be reduced to $\sim 0.7 \mathrm{eV}$.

Vertical Heterostructures. The vertical heterostructures are constructed by stacking $2 \mathrm{D} \mathrm{SL} \mathrm{MoTe} e_{2}$ and $\mathrm{NiTe}_{2}$ layers as described in Figure 1c,d. For a better understanding of heterostructures involving multilayer stacking of constituents, we first consider the variation of the electronic structures of $\mathrm{NiTe}_{2}$ and $\mathrm{MoTe}_{2}$ in SL (or monolayer), bilayer (BL), trilayer (TL), and periodic multilayer (ML) bulk forms. In forming bilayer, trilayer, and multilayer structures, interlayer distance and stacking geometry are fully optimized including vdW interaction. We found that $\mathrm{NiTe}_{2}$ remained metallic in SL and ML forms. However, the situation is critical for the semiconducting $\mathrm{MoTe}_{2}$ as shown in Figure 5. The monolayer structure of $\mathrm{MoTe}_{2}$ is a semiconductor with a direct band gap of $1.15 \mathrm{eV}$ occurring at the K-point of hexagonal BZ. In the bilayer, the band gap is slightly reduced and becomes indirect. It occurs between the maximum of the valence band at the $\Gamma$ point and the minimum of the conduction band along the $\Gamma-K$ direction. By stacking one more layer in TL, the gap continues to reduce, but remains indirect. Finally, in 3D layered $\mathrm{MoTe}_{2}$ the band gap is indirect and saturates at $0.75 \mathrm{eV}$. Apparently, the fundamental band gap of $\mathrm{MoTe}_{2}$ slabs reduces with an increasing number of layers and changes from direct to indirect. Our results are in fair agreement with the band gaps deduced from monolayers and multilayers of $\mathrm{MoTe}_{2}$. These results may have important implications in the context of band tunability which reflects the electronic properties of vertical heterostructures.

In a vertical heterostructure, $\mathrm{V}:\left(\mathrm{MoTe}_{2}\right)_{p} /\left(\mathrm{NiTe}_{2}\right)_{q}, p$ and $q$ are crucial parameters, which control the electronic properties. Confinements leading to electronic phase separation can take place for large $p=q \geq 2$. Additionally, as revealed in the above discussion, the electronic properties of the $\mathrm{MoTe}_{2}$ side depend

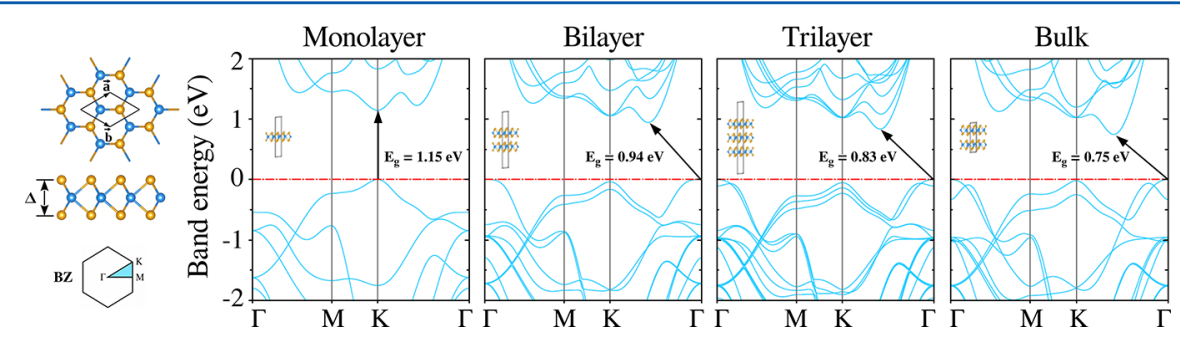

Figure 5. Electronic energy band structure of monolayer (SL), bilayer, trilayer, and periodic bulk $\mathrm{MoTe}_{2}$. The zero of energy is set at the top of the valence band. 
also on $p$. For the sake of comparison with lateral heterostructures, we first consider V: $\left(\mathrm{MoTe}_{2}\right)_{1} /\left(\mathrm{NiTe}_{2}\right)_{1}$ where alternatingly SL $\mathrm{MoTe}_{2}$ and $\mathrm{NiTe}_{2}$ are stacked. While this stacking is reminiscent of lateral and metallic A: $\left(\mathrm{MoTe}_{2}\right)_{1} /$ $\left(\mathrm{NiTe}_{2}\right)_{1}$, in the vertical one, as shown in Figure 6a, metallic

(a)

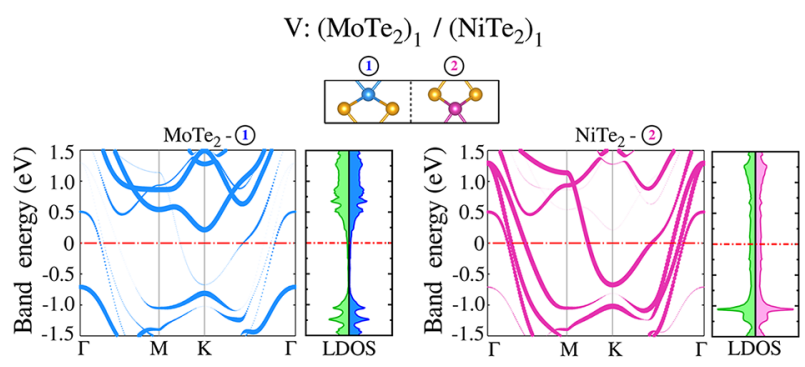

(b)

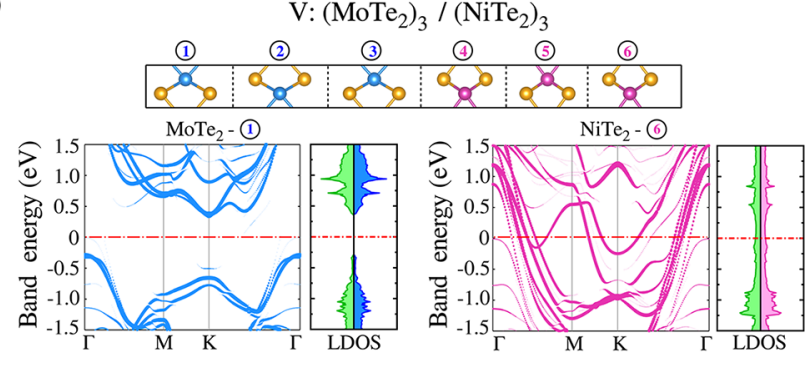

(c)

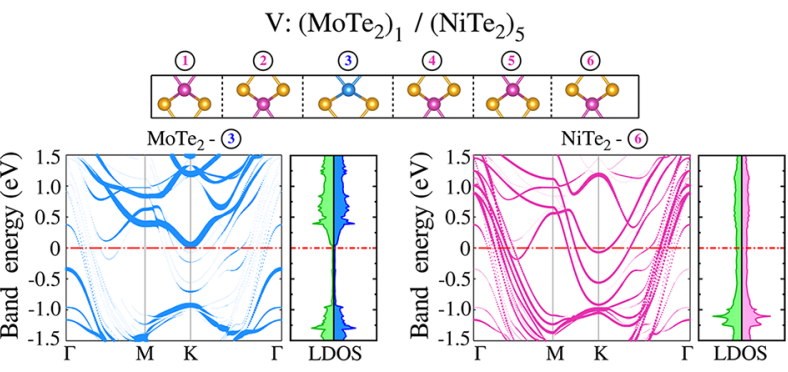

(d)

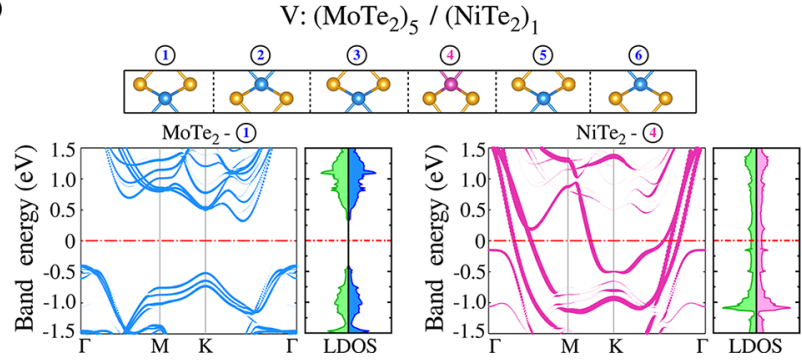

Figure 6. (a) V: $\left(\mathrm{MoTe}_{2}\right)_{1} /\left(\mathrm{NiTe}_{2}\right)_{1}$ heterostructure. Unit cell, energy bands, and corresponding state densities projected to adjacent Mo$\mathrm{Te}_{2}$ and $\mathrm{Ni}-\mathrm{Te}_{2}$ pairs. (b) $\mathrm{V}$ : $\left(\mathrm{MoTe}_{2}\right)_{3} /\left(\mathrm{NiTe}_{2}\right)_{3}$ heterostructure with a well-defined metal-insulator junction. (c) V: $\left(\mathrm{MoTe}_{2}\right)_{1} /\left(\mathrm{NiTe}_{2}\right)_{5} . \delta$ Doping of $\mathrm{NiTe}_{2}$ slab by a single sheet of $\mathrm{MoTe}_{2}$. (d) $\mathrm{V}:\left(\mathrm{MoTe}_{2}\right)_{5} /$ $\left(\mathrm{NiTe}_{2}\right)_{1} . \delta$-Doping of semiconducting $\mathrm{MoTe}_{2}$ slab by a single sheet of metallic $\mathrm{NiTe}_{2}$.

and semiconducting regions start to be separated in the direct space and acquire only minute state density at the $\mathrm{MoTe}_{2}$ unit. Apparently, the weak vdW interaction between adjacent SL $\mathrm{MoTe}_{2}$ and $\mathrm{NiTe}_{2}$ layers cannot couple the states of these two layers. In Figure $6 \mathrm{~b}$, the projected bands and LDOS of V: $\left(\mathrm{MoTe}_{2}\right)_{3} /\left(\mathrm{NiTe}_{2}\right)_{3}$ indicate that a well-defined metal-semiconductor junction is formed: the $\mathrm{MoTe}_{2}$ side appears as a semiconductor with an indirect band gap of $0.64 \mathrm{eV}$, while the $\mathrm{NiTe}_{2}$ side remains metallic in Figure 6b. This feature of vertical vdW heterostructures becomes even more critical in $\delta$ doping, where one SL TMD can be implemented in the thick slab constructed by the other SL TMD. In Figure 6c, an SL $\mathrm{MoTe}_{2}$ is placed on top of every vertically stacked five SL $\mathrm{NiTe}_{2}$ 's to form $\mathrm{V}:\left(\mathrm{MoTe}_{2}\right)_{1} /\left(\mathrm{NiTe}_{2}\right)_{5}$. This construction results in one layer of $2 \mathrm{D}$ semimetal in a thick metallic $\mathrm{NiTe}_{2}$ slab. Accordingly, the vertical conductivity of such a device is limited while the lateral $2 \mathrm{D}$ conductivity remains high. Conversely, in $\mathrm{V}:\left(\mathrm{MoTe}_{2}\right)_{5} /\left(\mathrm{NiTe}_{2}\right)_{1}$, a strictly $2 \mathrm{D}$ sheet of metallic zone is generated in a semiconducting slab having a band gap of $0.72 \mathrm{eV}$. Electrons confined in this $2 \mathrm{D}$ metallic sheet may exhibit interesting dimensionality effects and quantizations. $^{57-61}$ In particular, the conductance $G$ is quantized; it changes in steps of quantum of conductance with changes in Fermi energy; each band dipping in the Fermi level opens a conduction channel. Although it is not resolved from LDOS of $\delta$-doped $\mathrm{NiTe}_{2}$, the state density projected to the $2 \mathrm{D}$ metallic layer shows a staircase behavior in steps of $\mathrm{m} /$ $\pi h^{2}$ per unit area. ${ }^{62}$ Also, specific bands projected to $\mathrm{NiTe}_{2}$ in Figure $6 \mathrm{~d}$ dip into the Fermi level and increase the electron density under compressive strain. Eventually, conductance $G_{\|}$ jumps by the quantized value, $2 e^{2} / h$. Such a behavior can be used as a device function.

Crucial differences are observed between the vertical heterostructure $\mathrm{V}:\left(\mathrm{MoTe}_{2}\right)_{4} /\left(\mathrm{NiTe}_{2}\right)_{4}$ in Figure 7 and the lateral in-plane heterostructure A: $\left(\mathrm{MoTe}_{2}\right)_{10} /\left(\mathrm{NiTe}_{2}\right)_{10}$ in Figure 4, which become apparent in the electronic potential energy diagrams. For example, in the electronic potential energy diagram of the vertical heterostructures, one recognizes high potential energy regions between the adjacent layers. Due to the latter, the conduction phenomena in vertical heterostructures may become directional. The DOS projected to Te-cation-Te units shown in Figure $7 \mathrm{c}$ distinguishes two domains in the heterostructure. In the stack of $\mathrm{MoTe}_{2}$ the empty conduction band states are separated from filled valence band states by a fundamental band gap of $0.66 \mathrm{eV}$. In contrast, the DOS projected to $\mathrm{Ni}-\mathrm{Te}_{2}$ pairs are all metallic in the adjacent stack of four $\mathrm{NiTe}_{2}$ layers. This behavior indicates a periodic metal-semiconductor junction. Similar behavior occurs also in a nonperiodic single junction. The energy band diagram can be constructed in the direct space by joining conduction and valence band edges of each $\mathrm{Te}-\mathrm{Mo}-\mathrm{Te}$ unit. Here the interface between metallic and semiconducting regions is sharp. Since the charge transfer from the $\mathrm{MoTe}_{2}$ side to the $\mathrm{NiTe}_{2}$ side is minute in the absence of dopants near the interface layers, the bending or tilting of bands in direct space is negligible. However, three features are of importance: (i) At the $\mathrm{MoTe}_{2}$ side the band gap is slightly reduced at the interface. This is in compliance with the recent predictions. ${ }^{63}$ (ii) The largest gap at the $\mathrm{MoTe}_{2}$ side is smaller than the band gap of 2D SL $\mathrm{MoTe}_{2}$. This is in compliance with Figure 5. The energy bands projected to $\mathrm{Mo}-\mathrm{Te}_{2}$ and $\mathrm{Ni}-\mathrm{Te}_{2}$ units at different locations of the unit cell show the formation of semiconductor-metal junction with a Schottky barrier of $E_{\mathrm{S}}=$ $0.3 \mathrm{eV}$. (iii) Spin-polarized LDOSs are symmetric, and the whole system is nonmagnetic. It should also be noted the bands are expected to bend down in the case of n-type $\mathrm{MoTe}_{2}$ stack, where excess carriers spill to $\mathrm{NiTe}_{2}$.

\section{DISCUSSION AND CONCLUSIONS}

In this study our calculations reveal that periodic lateral and vertical heterostructures formed by semiconducting SL $\mathrm{MoTe}_{2}$ and metallic SL $\mathrm{NiTe}_{2}$ constitute two different class of 


$$
\mathrm{V}:\left(\mathrm{MoTe}_{2}\right)_{4} /\left(\mathrm{NiTe}_{2}\right)_{4}
$$

(a)

\begin{tabular}{|c|c|c|c|c|c|c|c|}
\hline (1) & (2) & (3) & (4) & (3) & (6) & (7) & (8) \\
\hline$b_{0}$ & & $d$ & 8 & & & & 18 \\
\hline
\end{tabular}

(b)

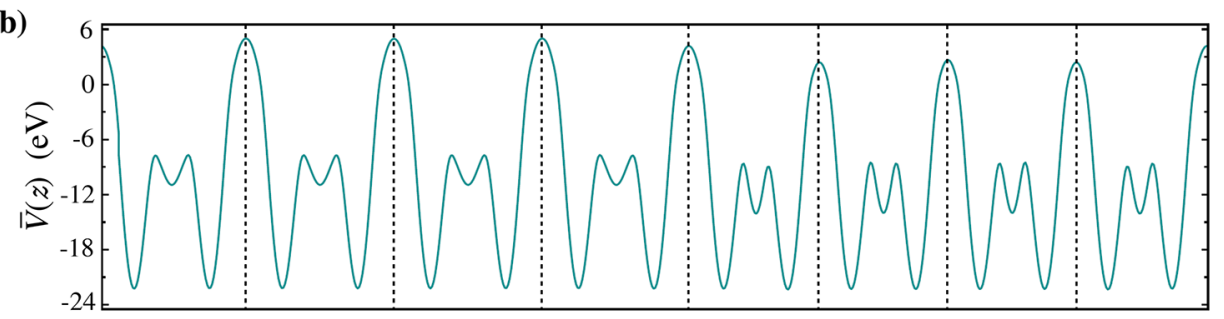

(c)

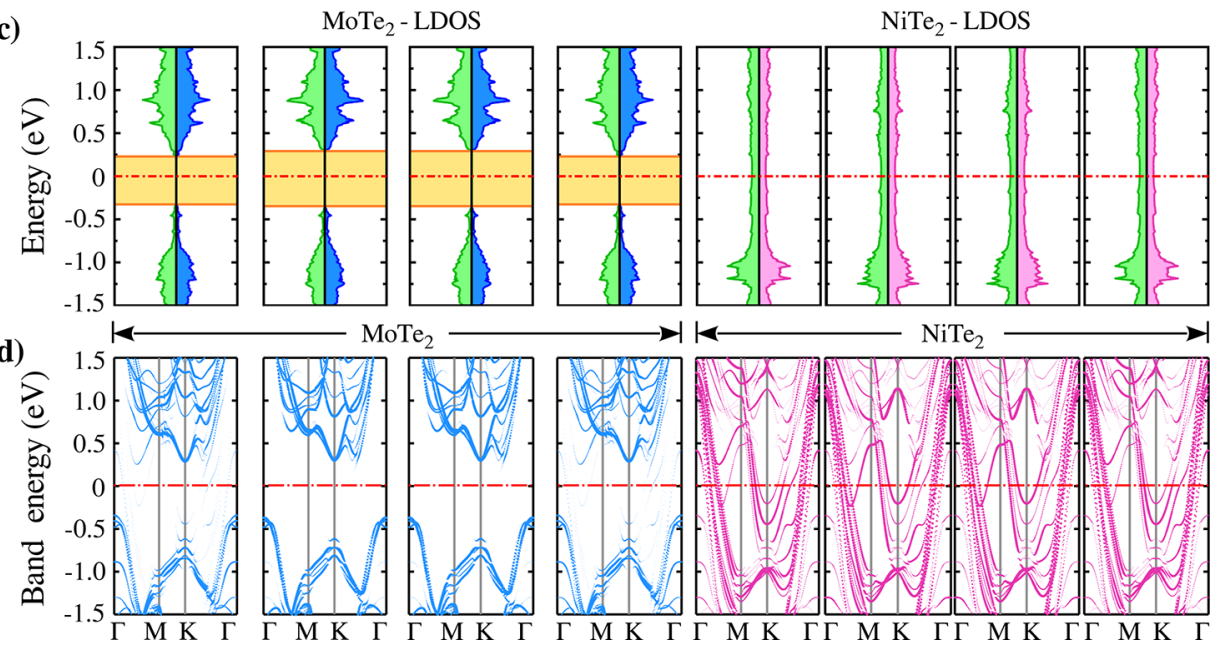

Figure 7. V: $\left(\mathrm{MoTe}_{2}\right)_{4} /\left(\mathrm{NiTe}_{2}\right)_{4}$ heterostructure. (a) The unit cell and chalcogen-cation-chalcogen units are specified by numerals. (b) Plane averaged electronic potential $\bar{V}(z)$. (c) Densities of states projected to chalcogen-cation-chalcogen units. The fundamental band gap at MoTe $e_{2}$ side is shaded. (d) Bands projected to chalcogen-cation-chalcogen units.

composite materials exhibiting diverse electronic properties depending on the composition (extension) of the constituents. The properties predicted in the present paper herald novel artificial materials, which can be crucial for $2 \mathrm{D}$ electronics.

In armchair edged, in-plane, lateral heterostructures, the coupling between different constituents is strong because of covelant + ionic $\mathrm{Mo}-\mathrm{Te}$ and $\mathrm{Ni}-\mathrm{Te}$ bonds crossing the boundaries. For small $p$ and $q$, namely for narrow stripes of constituents, the heterostructure is a composite metal. Upon widening of stripes, $p \geq 5$, a band gap is opened in the $\mathrm{MoTe}_{2}$ side. Electrons of metallic $\mathrm{NiTe}_{2}$ having energies coinciding with the band gap decay into the semiconducting side and pin the Fermi level. This way the metallic region penetrates the semiconductor and charge can transfer across the boundaries between two constituents. The boundaries have finite width, but are symmetric. The average electronic potential energy, which is rather flat and horizontal in the stripes, changes linearly across the boundary; no sawtooth-like tilting occurs. For finite size semiconducting stripes the band edges can conveniently be retrieved from the density of states projected to the chalcogen-metal-chalcogen units in the unit cell. This way, confinement effects are taken into account. For wide stripes, one can attain the fundamental band gap of 2D SL $\mathrm{MoTe}_{2}$. Energies of band edges relative to the average potential of SL $2 \mathrm{D} \mathrm{MoTe}{ }_{2}$ should coincide with those obtained directly from the LDOS at the center of the wide stripe. Our calculations indicate that the band gap is not uniform, but it is closed in the vicinity of the boundary. According to earlier methods developed initially for 3D heterostructures, Schottky barrier energy would be determined as the energy from the Fermi level to the edge of the conduction band, which follows the electronic potential energy $\bar{V}(z)$ and bends as shown by the dashed curve in Figure 4d. However, we found that band edges traced this way in the direct space deviate near the boundary from the ones determined directly using LDOS. This situation constitutes the dramatic effect of dimensionality.

Vertical heterostructures obtained by the periodic stacking of constituent 2D $\mathrm{MoTe}_{2}$ and $\mathrm{NiTe}_{2}$ layers behave rather differently from the armchair edged lateral heterostructure. The van der Waals interaction, albeit weak, is the main cause of the differences. The vertical heterostructure with $p=q=1$ exhibits semimetallic character along the $z$-direction with minute state density at the Te-Mo-Te unit, which is a good metal in the $(x y)$-plane. For $p=q \geq 2$ semiconducting and metallic phases are separated and metal-semiconductor junction is formed. The interface is sharp and planar, band bending is negligible for undoped $\mathrm{MoTe}_{2}$ stacks. The plane averaged electronic potential, $\bar{V}(z)$, is relatively high between two adjacent layers, which may limit the conductivity along the $z$-direction. The Schottky barrier is calculated as the energy from the Fermi level to the conduction band edge. The fundamental band gap at the semiconductor side is narrower as compared to lateral heterostructure, since the band gap of $\mathrm{MoTe}_{2}$ decreases and changes from direct to indirect by going from monolayer to bilayer and multilayers.

The $\delta$-doping is even more interesting in vertical structures. We predict that, by replacing one sheet of $\mathrm{NiTe}_{2}$ multilayer slab with $\mathrm{MoTe}_{2}$ monolayer, the structure acquires directional 
features: Along the $z$-direction it is a semimetal with the conduction band of $\mathrm{MoTe}_{2}$ touching the Fermi level and hence has poor perpendicular conductivity, $\sigma_{\perp}$, but it is a good metal and has good lateral conductivity $\sigma_{\|}$in the $\mathrm{NiTe}_{2}$ slab. Conversely, by replacing one sheet of the $\mathrm{MoTe}_{2}$ slab by $\mathrm{NiTe}_{2}$ monolayer, one can attain a strictly $2 \mathrm{D}$ metallic sheet within a semiconductor. Staircase-like densities of states and quantized conductance and also other features related to the dimensionality can be attained and exploited in 2D device applications.

In conclusion, 2D sheets of semiconducting and metallic TMDs stacked vertically and held by van der Waals interaction form vertical heterostructures that display features at variance with the lateral heterostructures constructed from the stripes of the same TMDs joined commensurately along their armchair edges. However, a Schottky barrier develops in both types of heterostructures when electrons become confined in different TMDs having sufficient size, i.e., width or thickness. It is demonstrated that by varying the size of constituents one can control the electronic structure and attain diverse properties. While the present study considered periodic arrangements of two constituents, finite arrangements or conformers with diverse size and orders of constituents can also result in crucial electronic devices.

\section{ASSOCIATED CONTENT}

\section{S Supporting Information}

The Supporting Information is available free of charge on the ACS Publications website at DOI: 10.1021/acs.jpcc.7b08256.

Crystallographic information for lateral and vertical heterostructures of transition metal dichalcogenides (ZIP)

Optimized structural parameters and calculated cohesive and formation energies and band gaps (PDF)

\section{AUTHOR INFORMATION}

\section{Corresponding Authors}

*E-mail: cetin_kilic@gtu.edu.tr.

*E-mail: ciraci@fen.bilkent.edu.tr.

\section{ORCID}

Çetin Kılıç: 0000-0003-2690-4940

\section{Notes}

The authors declare no competing financial interest.

\section{ACKNOWLEDGMENTS}

The calculations reported were performed at the High Performance and Grid Computing Center (TRUBA Resources) of TUBITAK ULAKBIM. S.C. acknowledges financial support from the Academy of Sciences of Turkey (TUBA).

\section{REFERENCES}

(1) Zhao, J.; Cheng, K.; Han, N.; Zhang, J. Growth Control, Interface Behavior, Band Alignment, and Potential Device Applications of 2D Lateral Heterostructures. WIREs Comput. Mol. Sci. 2017, e1353.

(2) Pradhan, N. R.; Talapatra, S.; Terrones, M.; Ajayan, P. M.; Balicas, L. Optoelectronic Properties of Heterostructures: The Most Recent Developments Based on Graphene and Transition-Metal Dichalcogenides. IEEE Nanotechnol. Mag. 2017, 11, 18-32.

(3) Zhang, Z.; Chen, P.; Duan, X.; Zang, K.; Luo, J.; Duan, X. Robust Epitaxial Growth of Two-Dimensional Heterostructures, Multiheterostructures, and Superlattices. Science 2017, 357, 788-792.

(4) Chiu, M.-H.; Li, M.-Y.; Zhang, W.; Hsu, W.-T.; Chang, W.-H.; Terrones, M.; Terrones, H.; Li, L.-J. Spectroscopic Signatures for
Interlayer Coupling in $\mathrm{MoS}_{2}-\mathrm{WSe}_{2}$ van der Waals Stacking. ACS Nano 2014, 8, 9649-9656.

(5) Paul, A. K.; Kuiri, M.; Saha, D.; Chakraborty, B.; Mahapatra, S.; Sood, A. K.; Das, A. Photo-Tunable Transfer Characteristics in $\mathrm{MoTe}_{2}-\mathrm{MoS}_{2}$ Vertical Heterostructure. NPJ. 2D Mater. Appl. 2017, 1, 17.

(6) Xue, Y.; Zhang, Y.; Liu, Y.; Liu, H.; Song, J.; Sophia, J.; Liu, J.; Xu, Z.; Xu, Q.; Wang, Z.; et al. Scalable Production of a Few-Layer $\mathrm{MoS}_{2}$ / $\mathrm{WS}_{2}$ Vertical Heterojunction Array and Its Application for Photodetectors. ACS Nano 2016, 10, 573-580.

(7) Gong, Y.; Lin, J.; Wang, X.; Shi, G.; Lei, S.; Lin, Z.; Zou, X.; Ye, G.; Vajtai, R.; Yakobson, B. I.; et al. Vertical and In-plane Heterostructures from $\mathrm{WS}_{2} / \mathrm{MoS}_{2}$ Monolayers. Nat. Mater. 2014, $13,1135-1142$.

(8) Chen, K.; Wan, X.; Wen, J.; Xie, W.; Kang, Z.; Zeng, X.; Chen, H.; Xu, J.-B. Electronic Properties of $\mathrm{MoS}_{2}-\mathrm{WS}_{2}$ Heterostructures Synthesized with Two-Step Lateral Epitaxial Strategy. ACS Nano 2015, 9, 9868-9876.

(9) Gong, Y.; Lei, S.; Ye, G.; Li, B.; He, Y.; Keyshar, K.; Zhang, X.; Wang, Q.; Lou, J.; Liu, Z.; et al. Two-Step Growth of TwoDimensional $\mathrm{WSe}_{2} / \mathrm{MoSe}_{2}$ Heterostructures. Nano Lett. 2015, 15, 6135-6141.

(10) Ross, J. S.; Rivera, P.; Schaibley, J.; Lee-Wong, E.; Yu, H.; Taniguchi, T.; Watanabe, K.; Yan, J.; Mandrus, D.; Cobden, D.; et al. Interlayer Exciton Optoelectronics in a $2 \mathrm{D}$ Heterostructure $\mathrm{p}-\mathrm{n}$ Junction. Nano Lett. 2017, 17, 638-643.

(11) Son, Y.; Li, M.-Y.; Cheng, C.-C.; Wei, K.-H.; Liu, P.; Wang, Q. H.; Li, L.-J.; Strano, M. S. Observation of Switchable Photoresponse of a Monolayer $\mathrm{WSe}_{2}-\mathrm{MoS}_{2}$ Lateral Heterostructure via Photocurrent Spectral Atomic Force Microscopic Imaging. Nano Lett. 2016, 16, 3571-3577.

(12) Duan, X.; Wang, C.; Shaw, J. C.; Cheng, R.; Chen, Y.; Li, H.; Wu, X.; Tang, Y.; Zhang, Q.; Pan, A.; et al. Lateral Epitaxial Growth of Two-Dimensional Layered Semiconductor Heterojunctions. Nat. Nanotechnol. 2014, 9, 1024-1030.

(13) Li, H.; Li, P.; Huang, J.-K.; Li, M.-Y.; Yang, C.-W.; Shi, Y.; Zhang, X.-X.; Li, L.-J. Laterally Stitched Heterostructures of Transition Metal Dichalcogenide: Chemical Vapor Deposition Growth on Lithographically Patterned Area. ACS Nano 2016, 10, 10516-10523.

(14) Chen, J.; Zhou, W.; Tang, W.; Tian, B.; Zhao, X.; Xu, H.; Liu, Y.; Geng, D.; Tan, S. J. R.; Fu, W.; et al. Lateral Epitaxy of Atomically Sharp $\mathrm{WSe}_{2} / \mathrm{WS}_{2}$ Heterojunctions on Silicon Dioxide Substrates. Chem. Mater. 2016, 28, 7194-7197.

(15) Yu, J. H.; Lee, H. R.; Hong, S. S.; Kong, D.; Lee, H.-W.; Wang, H.; Xiong, F.; Wang, S.; Cui, Y. Vertical Heterostructure of TwoDimensional $\mathrm{MoS}_{2}$ and $\mathrm{WSe}_{2}$ with Vertically Aligned Layers. Nano Lett. 2015, 15, 1031-1035.

(16) Li, M.-Y.; Shi, Y.; Cheng, C.-C.; Lu, L.-S.; Lin, Y.-C.; Tang, H.L.; Tsai, M.-L.; Chu, C.-W.; Wei, K.-H.; He, J.-H.; et al. Epitaxial Growth of a Monolayer $\mathrm{WSe}_{2}-\mathrm{MoS}_{2}$ Lateral $\mathrm{p}-\mathrm{n}$ Junction with an Atomically Sharp Interface. Science 2015, 349, 524-528.

(17) Yu, Y.; Hu, S.; Su, L.; Huang, L.; Liu, Y.; Jin, Z.; Purezky, A. A.; Geohegan, D. B.; Kim, K. W.; Zhang, Y.; et al. Equally Efficient Interlayer Exciton Relaxation and Improved Absorption in Epitaxial and Nonepitaxial $\mathrm{MoS}_{2} / \mathrm{WS}_{2}$ Heterostructures. Nano Lett. 2015, 15, 486-491.

(18) Li, C.; Yan, X.; Song, X.; Bao, W.; Ding, S.; Zhang, D. W.; Zhou, P. $\mathrm{WSe}_{2} / \mathrm{MoS}_{2}$ and $\mathrm{MoTe}_{2} / \mathrm{SnSe}_{2}$ van der Waals Heterostructure Transistors with Different Band Alignment. Nanotechnology 2017, 28, 415201.

(19) Terrones, H.; López-Urías, F.; Terrones, M. Novel HeteroLayered Materials with Tunable Direct Band Gaps by Sandwiching Different Metal Disulfides and Diselenides. Sci. Rep. 2013, 3, 1549.

(20) Kou, L.; Frauenheim, T.; Chen, C. Nanoscale Multilayer Transition-Metal Dichalcogenide Heterostructures: Band Gap Modulation by Interfacial Strain and Spontaneous Polarization. J. Phys. Chem. Lett. 2013, 4, 1730-1736. 
(21) Zhao, Y.-H.; Yang, F.; Wang, J.; Guo, H.; Ji, W. Continuously Tunable Electronic Structure of Transition Metal Dichalcogenides Superlattices. Sci. Rep. 2015, 5, 8356.

(22) Gu, K.; Yu, S.; Eshun, K.; Yuan, H.; Ye, H.; Tang, J.; Ioannou, D. E.; Xiao, C.; Wang, H.; Li, Q. Two-Dimensional Hybrid Layered Materials: Strain Engineering on the Band Structure of $\mathrm{MoS}_{2} / \mathrm{WSe}_{2}$ Hetero-Multilayers. Nanotechnology 2017, 28, 365202.

(23) Kośmider, K.; Fernández-Rossier, J. Electronic Properties of the $\mathrm{MoS}_{2}-\mathrm{WS}_{2}$ Heterojunction. Phys. Rev. B: Condens. Matter Mater. Phys. 2013, 87, 075451.

(24) Kang, J.; Tongay, S.; Zhou, J.; Li, J.; Wu, J. Band Offsets and Heterostructures of Two-Dimensional Semiconductors. Appl. Phys. Lett. 2013, 102, 012111.

(25) Wei, W.; Dai, Y.; Huang, B. In-plane Interfacing Effects of TwoDimensional Transition-Metal Dichalcogenide Heterostructures. Phys. Chem. Chem. Phys. 2016, 18, 15632-15638.

(26) Zhou, Y.; Dong, J.; Li, H. Multifunctional Heterostructures Constructed Using $\mathrm{MoS}_{2}$ and $\mathrm{WS}_{2}$ Nanoribbons. Phys. Chem. Chem. Phys. 2016, 18, 27468-27475.

(27) Cho, S.; Kim, S.; Kim, J. H.; Zhao, J.; Seok, J.; Keum, D. H.; Baik, J.; Choe, D.-H.; Chang, K. J.; Suenaga, K.; et al. Phase Patterning for Ohmic Homojunction Contact in $\mathrm{MoTe}_{2}$. Science 2015, 349, 625628.

(28) Katagiri, Y.; Nakamura, T.; Ishii, A.; Ohata, C.; Hasegawa, M.; Katsumoto, S.; Cusati, T.; Fortunelli, A.; Iannaccone, G.; Fiori, G.; et al. Gate-Tunable Atomically Thin Lateral $\mathrm{MoS}_{2}$ Schottky Junction Patterned by Electron Beam. Nano Lett. 2016, 16, 3788-3794.

(29) Aras, M.; Kılıç, Ç.; Ciraci, S. Planar Heterostructures of SingleLayer Transition Metal Dichalcogenides: Composite Structures, Schottky Junctions, Tunneling Barriers, and Half Metals. Phys. Rev. B: Condens. Matter Mater. Phys. 2017, 95, 075434.

(30) Fan, Z.-Q.; Jiang, X.-W.; Luo, J.-W.; Jiao, L.-Y.; Huang, R.; Li, S.S.; Wang, L.-W. In-plane Schottky-Barrier Field-Effect Transistors Based on $1 \mathrm{~T} / 2 \mathrm{H}$ Heterojunctions of Transition-Metal Dichalcogenides. Phys. Rev. B: Condens. Matter Mater. Phys. 2017, 96, 165402.

(31) Yang, Z.; Pan, J.; Liu, Q.; Wu, N.; Hu, M.; Ouyang, F. Electronic Structures and Transport Properties of a $\mathrm{MoS}_{2}-\mathrm{NbS}_{2}$ Nanoribbon Lateral Heterostructure. Phys. Chem. Chem. Phys. 2017, 19, 13031310

(32) Liu, Y.; Stradins, P.; Wei, S.-H. Van der Waals MetalSemiconductor junction: Weak Fermi Level Pinning Enables Effective Tuning of Schottky Barrier. Sci. Adv. 2016, 2, e1600069.

(33) Pan, S.; Ceballos, F.; Bellus, M. Z.; Zereshki, P.; Zhao, H. Ultrafast Charge Transfer Between $\mathrm{MoTe}_{2}$ and $\mathrm{MoS}_{2}$ Monolayers. 2D Mater. 2017, 4, 015033.

(34) Coleman, J. N.; Lotya, M.; O’Neill, A.; Bergin, S. D.; King, P. J.; Khan, U.; Young, K.; Gaucher, A.; De, S.; Smith, R. J.; et al. TwoDimensional Nanosheets Produced by Liquid Exfoliation of Layered Materials. Science 2011, 331, 568-571.

(35) Ataca, C.; Şahin, H.; Ciraci, S. Stable, Single-Layer $\mathrm{MoX}_{2}$ Transition-Metal Oxides and Dichalcogenides in a Honeycomb-Like Structure. J. Phys. Chem. C 2012, 116, 8983-8999.

(36) Levendorf, M. P.; Kim, C.-J.; Brown, L.; Huang, P. Y.; Havener, R. W.; Muller, D. A.; Park, J. Graphene and Boron Nitride Lateral Heterostructures for Atomically Thin Circuitry. Nature 2012, 488, 627-632.

(37) Hong, X.; Kim, J.; Shi, S.-F.; Zhang, Y.; Jin, C.; Sun, Y.; Tongay, S.; Wu, J.; Zhang, Y.; Wang, F. Ultrafast Charge Transfer in Atomically Thin $\mathrm{MoS}_{2} / \mathrm{WS}_{2}$ Heterostructures. Nat. Nanotechnol. 2014, 9, 682686.

(38) Liu, Z.; Lin, Y.-C.; Warner, J. H.; Teng, P.-Y.; Yeh, C.-H.; Chiu, P.-W.; Iijima, S.; Suenga, K. Characterization of Graphene and Transition Metal Dichalcogenide at the Atomic Scale. J. Phys. Soc. Jpn. 2015, 84, 121005.

(39) Ceperley, D. M.; Alder, B. J. Ground State of the Electron Gas by a Stochastic Method. Phys. Rev. Lett. 1980, 45, 566-569.

(40) Perdew, J. P.; Burke, K.; Ernzerhof, M. Generalized Gradient Approximation Made Simple. Phys. Rev. Lett. 1996, 77, 3865-3868.
(41) Grimme, S.; Antony, J.; Ehrlich, S.; Krieg, H. A Consistent and Accurate Ab Initio Parametrization of Density Functional Dispersion Correction (DFT-D) for the 94 Elements H-Pu. J. Chem. Phys. 2010, $132,154104$.

(42) Kresse, G.; Furthmüller, J. Efficient Iterative Schemes for $\mathrm{Ab}$ Initio Total-Energy Calculations Using a Plane-Wave Basis Set. Phys. Rev. B: Condens. Matter Mater. Phys. 1996, 54, 11169-11186.

(43) Blöchl, P. E. Projector Augmented-Wave Method. Phys. Rev. B: Condens. Matter Mater. Phys. 1994, 50, 17953-17979.

(44) Kresse, G.; Joubert, D. From Ultrasoft Pseudopotentials to the Projector Augmented-Wave Method. Phys. Rev. B: Condens. Matter Mater. Phys. 1999, 59, 1758-1775.

(45) Monkhorst, H. J.; Pack, J. D. Special Points for Brillouin-Zone Integrations. Phys. Rev. B 1976, 13, 5188-5192.

(46) Ataca, C.; Topsakal, M.; Aktürk, E.; Ciraci, S. A Comparative Study of Lattice Dynamics of Three- and Two-Dimensional $\mathrm{MoS}_{2}$. J. Phys. Chem. C 2011, 115, 16354-16361.

(47) Chang, C.-H.; Fan, X.; Lin, S.-H.; Kuo, J.-L. Orbital Analysis of Electronic Structure and Phonon Dispersion in $\mathrm{MoS}_{2}, \mathrm{MoSe}_{2}, \mathrm{WS}_{2}$, and $\mathrm{WSe}_{2}$ Monolayers Under Strain. Phys. Rev. B: Condens. Matter Mater. Phys. 2013, 88, 195420.

(48) Ruppert, C.; Aslan, O. B.; Heinz, T. F. Optical Properties and Band Gap of Single- and Few-Layer $\mathrm{MoTe}_{2}$ Crystals. Nano Lett. 2014, $14,6231-6236$

(49) Heyd, J.; Scuseria, G. E.; Ernzerhof, M. Hybrid Functionals Based on a Screened Coulomb Potential. J. Chem. Phys. 2003, 118, 8207-8215.

(50) Krukau, A. V.; Vydrov, O. A.; Izmaylov, A. F.; Scuseria, G. E. Influence of the Exchange Screening Parameter on the Performance of Screened Hybrid Functionals. J. Chem. Phys. 2006, 125, 224106.

(51) Schottky, W. Halbleitertheorie der Sperrschicht. Naturwissenschaften 1938, 26, 843-843.

(52) Mott, N. F. Note on the Contact Between a Metal and an Insulator or Semiconductor. Math. Proc. Cambridge Philos. Soc. 1938, $34,568-572$.

(53) Dandrea, R. G.; Duke, C. B. Calculation of the Schottky Barrier Height at the $\mathrm{Al} / \mathrm{GaAs}(001)$ Heterojunction: Effect of Interfacial Atomic Relaxations. J. Vac. Sci. Technol., A 1993, 11, 848-853.

(54) Baroni, S.; Resta, R.; Baldereschi, A.; Peressi, M. In Spectroscopy of Semiconductor Microstructures; Fasol, G., Fasolino, A., Lugli, P., Eds.; Springer US: Boston, MA, 1989; pp 251-271.

(55) Heine, V. Theory of Surface States. Phys. Rev. 1965, 138, A1689-A1696.

(56) Tersoff, J. Schottky Barrier Heights and the Continuum of Gap States. Phys. Rev. Lett. 1984, 52, 465-468.

(57) Landauer, R. Electrical Transport in Open and Closed Systems. Z. Phys. B: Condens. Matter 1987, 68, 217-228.

(58) Büttiker, M. Role of Quantum Coherence in Series Resistors. Phys. Rev. B: Condens. Matter Mater. Phys. 1986, 33, 3020-3026.

(59) van Wees, B. J.; van Houten, H.; Beenakker, C. W. J.; Williamson, J. G.; Kouwenhoven, L. P.; van der Marel, D.; Foxon, C. T. Quantized Conductance of Point Contacts in a Two-Dimensional Electron Gas. Phys. Rev. Lett. 1988, 60, 848-850.

(60) Tekman, E.; Ciraci, S. Novel Features of Quantum Conduction in a Constriction. Phys. Rev. B: Condens. Matter Mater. Phys. 1989, 39, $8772-8775$.

(61) Tekman, E.; Ciraci, S. Ballistic Transport Through a Quantum Point Contact: Elastic Scattering by Impurities. Phys. Rev. B: Condens. Matter Mater. Phys. 1990, 42, 9098-9103.

(62) Sólyom, J. Fundamentals of the Physics of Solids: Vol. 3 - Normal, Broken-Symmetry, and Correlated Systems; Theoretical Solid State Physics: Vol. 3 - Interaction Among Electrons; Springer: Berlin, 2010; p 343.

(63) Winther, K. T.; Thygesen, K. S. Band Structure Engineering in Van der Waals Heterostructures via Dielectric Screening: the G $\Delta W$ Method. 2D Mater. 2017, 4, 025059. 